\title{
Flexibilidad cognoscitiva en niños escolares con antecedentes de hipotiroidismo congénito
}

Juan Antonio González Medrano, ${ }^{1}$ Carmen Sánchez Pérez, ${ }^{1,2}$ Gabriela Romero Esquiliano, ${ }^{1}$ Javier Velázquez Moctezuma ${ }^{1}$ e Ignacio Méndez Ramírez ${ }^{3}$

\section{Introducción}

La comprensión funcional del cerebro en el desarrollo infantil implica un proceso de adquisición de conocimientos y habilidades que pueden verse alteradas por agentes o factores de riesgo biológicos o medioambientales que alteren el proceso evolutivo; debido a esto es importante analizar su organización. El estudio de las relaciones cerebro-comportamiento es el objetivo central de la neuropsicología (Matute, 2012). El cerebro es el órgano rector de la actividad cognitiva; en él participan diversas estructuras asociadas a múltiples funciones, las más estudiadas actualmente son las funciones ejecutivas vinculadas a los lóbulos frontales (Flores y Ostrosky-Shejet, 2012).

Las funciones ejecutivas implican conductas complejas a partir de acciones simples que incluyen el procesamiento y codificación de la información. Estas funciones neurales vinculadas a

1 Universidad Autónoma Metropolitana.

2 Instituto Nacional de Pediatría.

3 Universidad Nacional Autónoma de México. 
zonas frontales se relacionan a su vez con la programación motriz, control de la atención y memoria (Flores y Ostrosky-Solís, 2008). Los procesos involucrados en las funciones ejecutivas incluyen la planeación, control conductual, flexibilidad cognoscitiva, memoria de trabajo y fluidez (Flores y Ostrosky-Solís, 2008).

La flexibilidad cognoscitiva (FC) es la capacidad de adaptación, ajuste y modificación de la respuesta conductual o mental ante demandas del medio ambiente; se reorganiza así el procesamiento de información (Fitzgibbon, Cragg y Carroll, 2014). En este ajuste se considera el análisis de las consecuencias de la conducta y el aprendizaje de los errores; función a la que se asocia el control inhibitorio que refleja el dominio sobre activaciones automáticas (Flores y Ostrosky-Shejet, 2012).

El test de clasificación de tarjetas de Wisconsin (Heaton et al., 1997) es un instrumento en la evaluación de la FC, memoria operativa y atención (Matute, 2012). Consiste en el uso de 128 tarjetas-respuestas que deben ser organizadas por categorías de color, forma o número. Se ha reportado que a mayor edad, mayor número de categorías y menor número de respuestas perseverativas y errores (Heaton et al., 1997).

En el presente estudio se empleó una tarea similar de la Evaluación Neuropsicológica Infantil (ENI) con 54 tarjetas-respuesta y tres estímulos adaptada a población escolar (Matute, Rosselli, Ardila y Ostrosky-Solís, 2007). La prueba evalúa la formación conceptual. La consigna es que el niño coloque la tarjeta respuesta frente al estímulo según considere su relación. Se considera un feedback "correcto" o "incorrecto" cada vez que coloca una tarjeta adecuada o inadecuadamente; esto permite en el sujeto modificar su pensamiento a través de habilidades de clasificación y organización. Los indicadores para su análisis son el número de tarjetas seleccionadas y empleadas por el sujeto, número de categorías, total de respuestas correctas (es la suma de respuestas positivas), total de errores (ocurre cuando una respuesta no es pareada con la tarjeta estímulo utilizando la categoría en curso), e incapacidad para mantener la organización (consiste en la presencia de un error después de realizar cinco respuestas correctas). Tres factores son considerados en las funciones ejecutivas presentes: 
la velocidad en la ejecución, la calidad en la ejecución (considerada a partir del número de errores) y la economía en la ejecución (Matute, 2012).

Los resultados de la evaluación se realizan mediante la conversión del puntaje natural a puntaje escalar y la ubicación del percentil en relación con su edad cronológica, para obtener la clasificación de la ejecución: extremadamente bajo, bajo, promedio bajo, promedio y por arriba del promedio (Matute, RosseIli, Ardila y Ostrosky-Solís, 2007).

\section{Problema}

El hipotiroidismo congénito (HC) es un defecto sistémico producto de la disminución de la actividad tisular de las hormonas tiroideas triyodotironina (T3) y tiroxina (T4) durante la vida fetal y a partir del primer trimestre de vida. El tratamiento consiste en reemplazar la deficiencia de hormona tiroidea. Las medidas actuales para su atención implican una detección, diagnóstico e intervención de inicio anterior a los 15 días de vida posnatal (AAP, 2006 y Vela-Amieva, 2004).

Álvarez et al. (1999) plantean que las áreas del cerebro que coordinan la inteligencia ejecutiva y perceptual están relacionadas con las hormonas tiroideas durante el primer año de vida. Los pacientes diagnosticados y tratados precozmente tienen mayores posibilidades de alcanzar un cociente intelectual (cI) normal. El Hc en niños se ha asociado a secuelas neurológicas prevalentes posterior al diagnóstico y tratamiento temprano posterior a la evaluación de tamiz metabólico, manifestaciones tempranas en alimentación, tono muscular y fisiológicas (Sánchez, 2006). Las secuelas tardías están presentes en la neurocognición en etapa escolar, retraso mental de grado variable, déficit de atención, incoordinación motora gruesa, presencia de hipertonía o hipotonía, problemas conductuales (CENETEC, 2008). Se han identificado algunos defectos en el procesamiento visoespacial, memoria selectiva y defectos sensoriomotores. Aijaz et al. (2006) reportan que a los ocho años los niños, con Hc con 
inicio de tratamiento temprano tratados antes de los tres meses de edad presentan déficit en la atención con ausencia de hiperactividad. El objetivo del presente trabajo es describir la flexibilidad cognoscitiva en niños con antecedente de hipotiroidismo congénito detectados por tamiz metabólico y tratados con hormona tiroidea, y asociarlo con el tipo de Hc y cociente intelectual (cl). Secciones (según sea reporte de investigación u otro tipo).

\section{Método}

Se consideró un diseño de estudio observacional, descriptivo, prospectivo y transversal. Participaron niños de edad escolar (8 y 9 años) con diagnóstico de Hc confirmado por perfil tiroideo en el servicio de endocrinología. Los datos se obtuvieron del expediente clínico hospitalario. Asisten desde la etapa del lactante a programa de seguimiento del neurodesarrollo en el Instituto $\mathrm{Na}$ cional de Pediatría (INP) y con inicio de tratamiento variable antes del tercer mes de vida extrauterina. El registro de la FC se realizó con la Evaluación Neuropsicológica Infantil (ENI) con la escala de funciones ejecutivas-flexibilidad cognoscitiva. El cl se obtuvo mediante escala de inteligencia infantil WISc-R. El análisis estadístico consistió en la descripción de variables categóricas con frecuencias y en la asociación entre variables el análisis consideró una $p$ $<0.05$ mediante prueba de chi² $^{2}$.

\section{Resultados}

En el análisis descriptivo participaron 33 niños, 17 de 8 años y 16 de 9 años; 6 niños de sexo masculino y 27 de sexo femenino; por tipo de hipotiroidismo, 19 casos de atirosis y 14 de ectopia; por edad de inicio de tratamiento se conformaron tres grupos: entre los 0 y 30 días 6 casos, entre los 31 y 60 días, 21 y entre los 61 y 90 días fueron 6 . En relación con el cı global: 2 discapacidad intelectual, 5 limítrofe, 7 normal torpe, 14 niños normal y 5 normal brillante; por cı ejecutivo, 2 discapacidad inte- 
lectual, 4 limítrofe, 7 normal torpe, 15 normal, 3 normal brillante y 2 superior; y en el cl verbal: 2 deficiente mental, 6 limítrofe, 8 normal torpe, 13 normal y 4 normal brillante.

En ambos grupos de edad se emplearon las 54 tarjetas respuesta para la evaluación de la flexibilidad cognitiva. A los 8 años prevalece el uso de las categorías de color (1 categoría), color y forma (2 categorías); a diferencia de los 9, donde el uso más frecuente fue de 2 categorías (color y forma); pocos casos llegan a emplear la categoría de número.

El número total de respuestas positivas/correctas en el grupo de 8 años es de 21, y en el de 9 años es de 25. En la incapacidad para la clasificación se registró que en ambos grupos los valores son los esperados para su edad.

Con respecto al total de errores, los niños de 8 años cometen más errores que los de 9 (33 y 28, respectivamente).

Los niños con ectopia tuvieron mayor número de respuestas positivas totales en comparación con los de atirosis $(p=.05)$, y en correspondencia menor número de errores en los niños con ectopia $(p=.02)$. No se asoció el Hc con el número de categorías.

El número total de categorías empleadas y la edad tuvieron una asociación marginal $p=.06$. El sexo se asoció con el número de categorías empleadas $(p=.03)$. La edad se asoció con el número de respuestas positivas totales $(p=0.02)$. No se encontró relación entre el cociente intelectual e inicio de tratamiento con la FC.

\section{Conclusiones}

Como era de esperarse, el Hc es más frecuente en mujeres que en hombres, con una relación de 2:1, situación observable en este estudio (Martínez y Orozco, 2008).

En los niños con antecedente de $\mathrm{Hc}$, el razonamiento abstracto y la habilidad para cambiar de estrategias están asociados a la edad (Soprano, 2009), conforme incrementa la edad el nivel de ejecución mejora en la disminución de errores.

Los niños que iniciaron tratamiento entre los 31 y 60 días tienen mejor diagnóstico en la Fc, calificando como promedio sin 
encontrar asociación estadísticamente significativa. En casos de HC, la sustitución adecuada de hormonas tiroideas es de gran importancia por el papel que juegan éstas en el crecimiento y desarrollo, especialmente en la diferenciación del sistema nervioso central (Rojas, 2009).

Ardila (2007) menciona que las pruebas de inteligencia no son lo suficientemente sensibles en el análisis de la organización de procesos cognoscitivos en los niños con $\mathrm{HC}$, donde no se encontró relación estadísticamente significativa entre el $\mathrm{Cl}$ y el HC.

La excesiva fijación de un criterio se relaciona con una hipótesis o una estrategia de acción, afectando de forma importante la solución de problemas (Flores, y Ostrosky-Solís, 2008).

La incapacidad para mantener la organización se relaciona con las respuestas perseverantes, en aquellos casos bajos y extremadamente bajos hay una menor flexibilidad cognoscitiva (Matute, 2012). En los niños con antecedente de Hc, la capacidad de modificar su foco de atención se encuentra dentro de los valores promedio; sin embargo, en la ejecución y resolución de la tarea se observa la dificultad de control inhibitorio y con ello en modificar las respuestas que ya no son requeridas, dicho proceso podría asociarse a problemas de atención.

El uso de instrumentaciones neuropsicológicas en el seguimiento del desarrollo en edad escolar de niños con antecedente de $\mathrm{HC}$ favorece el análisis y discusión de los procesos cognoscitivos.

\section{Referencias}

Aijaz, N.J. et al. (2006) Neurocognitive Function in Children with Compensated Hypothyroidism: Lack of Short Term Effects on or Off Thyroxin. вMc Endocr Disord, 6(2), 1-7.

Álvarez, M., Güell, R., Daniel, L., Berazaín, A.R., Machado, C. y Pascual, A. (1999). Estado neurocognitivo en niños de 8 años con hipotiroidismo congénito tratado precozmente, Rev Neurol, 28(7), 701-706. 
APP (2006). Update of Newborn Screening and Therapy for Congenital Hypothyroidism. Pediatrics, 117(2290), 2290-2303.

Ardila, A. y Rosselli, M. (2007). Neuropsicología clínica. México: Manual Moderno.

CENETEC (2008). Prevención, diagnóstico y tratamiento del hipotiroidismo congénito neonatal en el primer nivel de atención. México: Secretaría de Salud.

Fitzgibbon, L., Cragg, L. y Carroll, D.J. (2014). Primed to be Inflexible: the Influence of Set Sixe on Cognitive Flexibility During Childhood. Front Psychol, 12(5), 101.

Flores, J.C. y Ostrosky-Solís, F. (2008). Neuropsicología de los lóbulos frontales, funciones ejecutivas y conducta humana. Revista Neuropsicología, Neuropsiquiatría y Neurociencias, 8(1), 47-58.

Flores, J.C. y Ostrosky-Shejet, F. (2012). Desarrollo neuropsicológico de lóbulos frontales y funciones ejecutivas. México: Manual Moderno.

Heaton, R. et al. (1997). Test de clasificación de tarjetas de Wisconsin. Madrid: Ediciones TEA.

Martínez-Medina, M.A. y Orozco-Cárdenas, M.B. (2008). Hipotiroidismo congénito. Reporte de un caso diagnosticado por tamiz neonatal. Bol Clin Hosp Infant., 25(2), 115-118.

Matute, E. (2012). Tendencias actuales de las neurociencias cognitivas. México: Manual Moderno.

Matute, E., Rosselli, M., Ardila, A. y Ostrosky-Solís, F. (2007). Evaluación neuropsicológica infantil. México: Manual Moderno.

Portellano, J.A. (2005). Cómo desarrollar la inteligencia. Madrid: Colección Especiales Somos.

Rojas, A., Garrido, E. y Nishimura, E. (2009). Manejo del hipotiroidismo congénito con dosis diarias no equitativas de levotiroxina. Revista de Endocrinología y Nutrición, 17(2), 66-70.

Sánchez, M. et al. (2006). Hipotiroidismo congénito. Manifestaciones clínicas en niños menores de 15 semanas con tamiz neonatal positivo. Revista Mexicana de Pediatría, 73(6), 272-279. 
Soprano, A.M. (2009). Cómo evaluar la atención y las funciones ejecutivas en niños y adolescentes. Buenos Aires: Paidós.

Vela-Amieva, M. et al. (2004). Epidemiología del hipotiroidismo congénito en México. Salud Pública de México, 46(2), 211224. 